\title{
BMJ Open Patient-reported gout attack frequency and allopurinol use in general practice in the Netherlands: a prospective observational cohort study protocol
}

\author{
Kevin D B van Leeuwen, ${ }^{1}$ Arthur M Bohnen, ${ }^{1}$ Marloes L Jacobs, ${ }^{1}$ \\ Johan van Der Lei, ${ }^{2}$ Hein J E M Janssens, ${ }^{3}$ Aafke R Koffeman, ${ }^{4}$ \\ Patrick J E Bindels, ${ }^{1}$ Sita M A Bierma-Zeinstra ${ }^{1}$
}

To cite: van Leeuwen KDB, Bohnen AM, Jacobs ML, et al. Patient-reported gout attack frequency and allopurinol use in general practice in the Netherlands: a prospective observational cohort study protocol. BMJ Open 2018;8:e024335. doi:10.1136/ bmjopen-2018-024335

- Prepublication history for this paper is available online. To view these files, please visit the journal online (http://dx.doi org/10.1136/bmjopen-2018024335).

Received 22 May 2018 Revised 29 August 2018 Accepted 26 September 2018

Check for updates

(C) Author(s) (or their employer(s)) 2018. Re-use permitted under CC BY-NC. No commercial re-use. See rights and permissions. Published by BMJ.

${ }^{1}$ Department of General Practice, Erasmus Medical Center, Rotterdam, The Netherlands ${ }^{2}$ Department of Medical Informatics, Erasmus Medical Center, Rotterdam, Netherlands ${ }^{3}$ Department of General Practice, Radboud University Medical Center, Nijmegen, Netherlands ${ }^{4}$ Department of Elderly Health, Leids Universitair Medisch Centrum, Leiden, Netherlands

Correspondence to

Kevin D B van Leeuwen;

k.leeuwen@erasmusmc.n

\section{ABSTRACT}

Introduction Gout is the most common inflammatory arthritis in the Dutch general practice population and is often managed with long-term uric acid lowering treatment. The clinical relevance of this treatment in preventing gout attacks is unclear.

Primary research question What is the frequency of self-reported gout attacks and what is the effect of allopurinol use in patients diagnosed with gout in general practice?

Methods and analysis Adult patients with a diagnostic consultation code for gout in the year 2013, 2014 or 2015 will be invited to participate in this prospective observational cohort study. Patients with a limited life expectancy will be excluded. Baseline measurements will include blood pressure, body mass index and a blood sample (estimated glomerular filtration rate, serum uric acid, cholesterol (low-density lipoprotein (LDL) and high-density lipoprotein), glucose (fasting)). At the 2-year follow-up, patients will receive questionnaires every 3 months. The questionnaires at baseline, 12 months and 24 months assess the frequency of gout attacks, the presence of tophi, comorbidity, medication use, quality of life, diet and lifestyle. The questionnaires in between only assess the frequency of gout attacks and medication use for gout. Descriptive statistics will be used to calculate the mean frequency of self-reported gout attacks during the 2-year follow-up. The propensity score for each patient being offered allopurinol is estimated and used to match patients with and without allopurinol treatment. We will compare the frequency of gout attacks in these groups using multilevel Poisson regression analyses. With this type of analysis, we can calculate the corrected estimated effect of allopurinol on gout attack frequency.

Ethics and dissemination The research protocol was approved by the Medical Ethical Committee of the Erasmus Medical Centre in Rotterdam. The knowledge generated by this study will be transferred to the Dutch College of General Practitioners, conferences and to (inter)national peer-reviewed journals.

Trial registration number NTR6329; Pre-results.
Strengths and limitations of this study

- This study will recruit and follow-up patients in a large primary care population, providing important information about gout attack frequencies, risk factors and treatment of gout in general practice.

- Gout diagnosis is based on the interpretation of general practitioners, American College of Rheumatology/ European League Against Rheumatism criteria and the gout calculator rather than synovial fluid analysis for the presence of monosodium urate crystals, the current gold standard for gout diagnosis.

- Evaluating the effect of allopurinol treatment as prescribed in daily practice will result in a much higher generalisability and improve feasibility when compared with performing an randomised controlled trial.

- In our sample of patients with prevalent gout, there may be variation in treatment duration of allopurinol. If treatment duration of allopurinol is associated with the number of experienced gout attacks then adjustment or stratification for treatment duration is necessary.

\section{INTRODUCTION}

\section{Background and rationale}

Gout is the most common inflammatory arthritis, affecting $1.7 \%$ of the total Dutch general practice population, and $3 \%-4 \%$ of the adult population. ${ }^{1}$ Acute gout is one of the most painful forms of arthritis and is characterised by the abrupt onset of severe joint pain (classically the first metatarsophalangeal joint), swelling and erythema. It is caused by the deposition of monosodium uric acid crystals in and around joints, leading to an aseptic arthritis. The appearance of such crystal deposition is strongly associated with a raised serum uric acid concentration (hyperuricaemia). However, although there is a clear association between hyperuricaemia and gout, only a minority of people with hyperuricaemia actually go on to develop gouty 
arthritis. ${ }^{2}$ In practice, gout is often managed with (lifelong) uric acid-lowering medication, most often allopurinol. There is limited evidence that it actually reduces the gout attack frequency and/or resolves tophi if present, or prevents longterm joint damage. ${ }^{3}$ Randomised trials have only provided evidence on serum uric acid concentrations, a surrogate outcome, and not on clinical endpoints. ${ }^{3}$ In addition, there is much debate on the indications for, and best time to start with long-term uric acid lowering therapy. ${ }^{4}$ Although allopurinol treatment is recommended by American College of Rheumatology (ACR) and European League Against Rheumatism (EULAR) guidelines, recently the American College of Physicians recommended against initiating long-term urate-lowering therapy in patients with infrequent attacks. ${ }^{5}$ Other risk factors, which are traditionally thought to play a causal role in the development of gout include diet, overweight and use of medication, but the exact mechanisms by which these might lead to gout or might be associated with gout attack frequency are unclear. Recent studies show contradictory of inconclusive results. ${ }^{6-9}$

\section{Objectives}

The primary objective of this cohort study is to measure the frequency of self-reported gout attacks and the prevalence of tophi in patients diagnosed with gout in general practice, and to assess whether the use of allopurinol decreases the self-reported gout attack frequency and the prevalence of tophi.

Secondary objectives of this study are: (1) to assess whether patient characteristics and lifestyle factors (such as the presence of cardiovascular risk factors, body mass index (BMI), smoking status, physical activity and social status) are associated with the self-reported frequency of gout attacks; (2) to assess whether the consumption of fructose rich beverages, carbonated beverages, alcohol, purine rich food, lactose and dairy products are associated with the self-reported frequency of gout attacks; (3) to assess whether the use of medication, such as diuretics and salicylates, is associated with gout attack frequency.

\section{Study design}

Multicentre prospective observational cohort study of patients diagnosed with gout in general practice. The coordinating centre is the department of general practice, Erasmus Medical Centre.

\section{METHODS AND ANALYSIS}

\section{Study setting}

The study will be conducted in the primary care community in the Netherlands.

\section{Study population}

\section{Inclusion criteria}

Patients, 18 years or older, with at least one gout attack between January 2013 and December 2015 as diagnosed by his or her general practitioner (GP), will be screened for eligibility.
Exclusion criteria

Patients with a limited life expectancy and patients who (independently or with help) are not able to fill in the Dutch questionnaires will be excluded.

\section{Patient selection and recruitment}

Potential eligible patients will be anonymously selected from the Integrated Primary Care Information (IPCI) database: a longitudinal primary healthcare database. ${ }^{10}$ This database contains the electronic patient records of over 1.5 million patients registered with over 700 GPs throughout the Netherlands. It contains all journal entries by the GP, coded diagnoses using the International Classification of Primary Care (ICPC) codes, referrals, clinical findings by specialists, laboratory findings and hospitalisations. ${ }^{11}$ Episodes of gout will be identified based on ICPC-coding (T92) and free text (gout). The patient's identity in the IPCI-database is anonymised. However, all patients in the IPCI-database have a unique patient identity code which can only be decrypted by their GP. Every GP in the database has a unique GP identity code which can only be decrypted by the database gatekeeper. The gatekeeper will provide participating GPs with a list of unique identity codes of the selected patients. The GP is able to decrypt the code and will assess whether the patient is eligible according to the inclusion and exclusion criteria. Eligible patients will receive written information and an informed consent form from the GP. They will be requested to send their contact details to the research centre if they are willing to participate. A telephonic interview will take place to answer any questions the patient has, followed by the informed consent procedure. To improve the response rate, reminders will be sent to general practices and eligible patients.

\section{Sample size calculation}

With a significance level of 0.05 and a power of 0.99 , we are able to detect a difference in an average number of 2.8 attacks in the untreated and of 1.4 in the treated group when we include 227 patients using allopurinol and perform a 1:2 matching. Our explorative search in the IPCI database in 2016 indicated that about one-third of the patients diagnosed with gout by the GP use allopurinol. This would mean that we at least have to include 681 patients with gout (227 allopurinol users and 454 non-users). When the treatment effect is substantially lower with 2.8 in the untreated and 2.4 in the treated patients, we still have a power of 0.85 to detect this difference with the same level of significance. Equally, this high power allows exclusion of up to $40 \%$ non-matchable patients (in case of 1:2 matching and 2.8 vs 1.4 attacks in the different groups). Finally, to account for $10 \%$ potential loss to follow-up, we aim to include at least 757 patients.

\section{Study outcomes}

The outcomes are the frequency of self-reported gout attacks per year and the prevalence of tophi in patients diagnosed with gout in general practice.

\section{Data collection and research measures Clinical measurements}

At baseline, a blood sample and a physical examination will take place. The blood sample includes a laboratory 
examination of estimated glomerular filtration rate (eGFR), uric acid level, total cholesterol, low-density and high-density lipoprotein cholesterol and fasting glucose. All patients will be asked to visit their GP for a physical examination to obtain a standardised blood pressure (the average blood pressure of three measurements), and measurement of height and weight to calculate the BMI. These factors contribute to an approximation of the cardiovascular risk and general health.

\section{Patient demographics}

At baseline age, gender, marital status, level of education and current occupation will be recorded.

\section{Gout attacks}

In a baseline questionnaire, the presence of gout will be validated according to the 2015 ACR-EULAR criteria for gout and the diagnostic rule (Gout calculator). ${ }^{12} 13$ The date of the first gout attack, the lifetime number of gout attacks, characteristics of the most recent gout attack such as localisation and severity of the attack, and all medication use related to gout will be evaluated. These factors contribute to the indication for allopurinol treatment according to the Dutch primary care guidelines. ${ }^{1}$ The gout attack frequency (including localisation and severity of the most recent gout attack and all medication use related to gout and adherence) will be repeatedly evaluated every 3 months in follow-up questionnaires. In the questionnaires, a gout attack is defined as the time a joint is painful or swollen because of gout. To measure the disease-specific impact and consequences of gout, the validated Gout Assessment Questionnaire 2.0 will be used at baseline, and at 1-year and 2-year follow-ups. ${ }^{14-16}$

\section{Presence of tophi}

The patient-reported presence of tophi and the physical and mental burden of tophi will be determined at baseline, after 3 months, 1 year and 2 years follow-up, using the Tophus Impact Questionnaire-20. ${ }^{17}$ Explanatory text and images of tophi are presented in the questionnaire. The time point at 3 months follow-up was chosen to validate the questionnaire for the Dutch language, since this was not previously done. The time points at 1 and 2 years follow-up were chosen to measure changes in the presence and burden of tophi.

\section{Comorbidity}

Comorbidity will be assessed at baseline, 1year and 2 years follow-up using the Self-administered Comorbidity Questionnaire. ${ }^{18}$

\section{Physical and mental health}

Physical and mental health will be determined at baseline, 1year and 2years using the Short Form-36 physical and mental component summary and quality of life via the EuroQol 5 dimensions. ${ }^{19}{ }^{20}$ Physical activity will be measured with the International Physical Activity Questionnaire. $^{21}$
Medication use

The use of all prescribed and over-the-counter medication will be measured at baseline, 1 year and 2 years follow-up using a derived version of the Brief Medication Questionnaire. $^{22}$

\section{Diet and smoking}

Dietary intake will be measured using the Dutch Food Frequency Questionnaire. ${ }^{23-26}$ This validated questionnaire inventories all dietary habits over the past month and will be administered at baseline, 1 and 2 years follow-up. Smoking status will be asked every year.

All data collection and time points are pictured in table 1. A patient time line is presented in figure 1.

The questionnaires will be electronically mailed to the patients using GemsTracker (GEneric Medical Survey Tracker). This is a software package for (complex) distribution of questionnaires and forms during clinical research and quality registrations in healthcare. Patients not able to work with a computer receive the questionnaires on paper. The deadline of each questionnaire is 4 weeks. Patients are reminded after 1 week (email), 2 weeks (telephone call) and 3 weeks (email). If a questionnaire is not filled out, it is recorded as missing.

\section{Data analysis}

The frequency of gout attacks and the prevalence of tophi will be calculated by means of descriptive statistics. To investigate the association between the use of allopurinol and the frequency of self-reported gout attacks, and respectively, the presence of tophi, we will use Poisson regression analyses, adjusted and unadjusted for risk factors for attack frequency. Before Poisson regression analysis, we will apply a propensity score matching analysis with calliper matching. First, the conditional probability that the patient will be offered allopurinol treatment will be estimated for each patient, based on a number of important factors. To identify these factors, binary logistic regression analyses will be conducted with allopurinol treatment (yes/no) as dependent and each potential confounder as independent variable. Selected potential confounders (expected to be related to the severity of gout) are: the presence of tophi, renal function (eGFR), blood pressure at baseline, time since gout diagnosis and the previous number of gout attacks per year. Other potential confounders are: age, gender, social status, baseline uric acid level (above or below target level of $0.35 \mathrm{mmol} / \mathrm{L}$ ), BMI, smoking behaviour, physical activity, presence of comorbidity and consumption of alcohol and purine rich food. Variables with a $p$ value smaller than 0.10 will be selected for the estimation of the propensity score. Subsequently, the derived propensity scores will be used for one-to-many matching, where patients with allopurinol treatment are matched to as many patients as possible without allopurinol treatment. These matched groups will be selected using nearest neighbour matching, with no replacement. To ensure that all matching will be adequately similar, an additional 
Table 1 Summary of data collection and timeline

\begin{tabular}{|c|c|c|c|c|c|c|c|c|c|}
\hline \multirow[b]{2}{*}{ Data collection } & \multicolumn{9}{|c|}{ Timeline (months from baseline) } \\
\hline & 0 & 3 & 6 & 9 & 12 & 15 & 18 & 21 & 24 \\
\hline $\begin{array}{l}\text { Physical examination (blood } \\
\text { pressure, height, weight) }\end{array}$ & $x$ & & & & & & & & \\
\hline $\begin{array}{l}\text { Laboratory serum examination: } \\
\text { eGFR, uric acid level, TC, LDL, HDL, } \\
\text { glucose }\end{array}$ & $x$ & & & & & & & & \\
\hline $\begin{array}{l}\text { Gout attacks: frequency, } \\
\text { characteristics, medication use and } \\
\text { adherence (GAQ) }\end{array}$ & $x$ & $\mathrm{X}$ & $x$ & $x$ & $x$ & $x$ & $x$ & $x$ & $x$ \\
\hline $\begin{array}{l}\text { Presence and burden of tophi (TIQ- } \\
\text { 20) }\end{array}$ & $x$ & $x$ & & & $x$ & & & & $\mathrm{X}$ \\
\hline Quality of life (EQ-5D) & $x$ & & & & $x$ & & & & $\mathrm{X}$ \\
\hline Medication use (general) (BMQ) & $x$ & & & & $x$ & & & & $x$ \\
\hline Diet (FFQ) and smoking status & $\mathrm{X}$ & & & & $x$ & & & & $X$ \\
\hline
\end{tabular}

$\mathrm{BMQ}$, Brief Medication Questionnaire; eGFR, estimated glomerular filtration rate; EQ-5D, EuroQol 5 Dimensions; FFQ, Food Frequency Questionnaire; GAQ, Gout Assessment Questionnaire; HDL, high-density lipoprotein cholesterol; IPAQ, International Physical Activity Questionnaire; LDL, low-density lipoprotein cholesterol; SCQ, Self-administered Comorbidity Questionnaire; SF-36, Short Form health survey; TC, total cholesterol; TIQ-20, Tophus Impact Questionnaire 20.

restriction, known as calliper matching, will be imposed to ensure that the propensity scores of control patients (without allopurinol treatment) lie within a prespecified interval of the propensity score of their matched case. All patients without appropriate matched patients in the other group will be excluded from the matched sample. To assess whether the matched sample is balanced, the characteristics of both groups will be compared before and after propensity score matching. Then, a corrected estimated effect of allopurinol will be calculated using a multilevel Poisson regression analysis, in which the propensity score is used as a matching variable. Potential confounders will be added to the regression model, such as time from first allopurinol use and serum urate level at baseline.

To answer the secondary objectives, associations will be estimated using multivariable logistic regression analysis. The dependent variable will be patients' reported

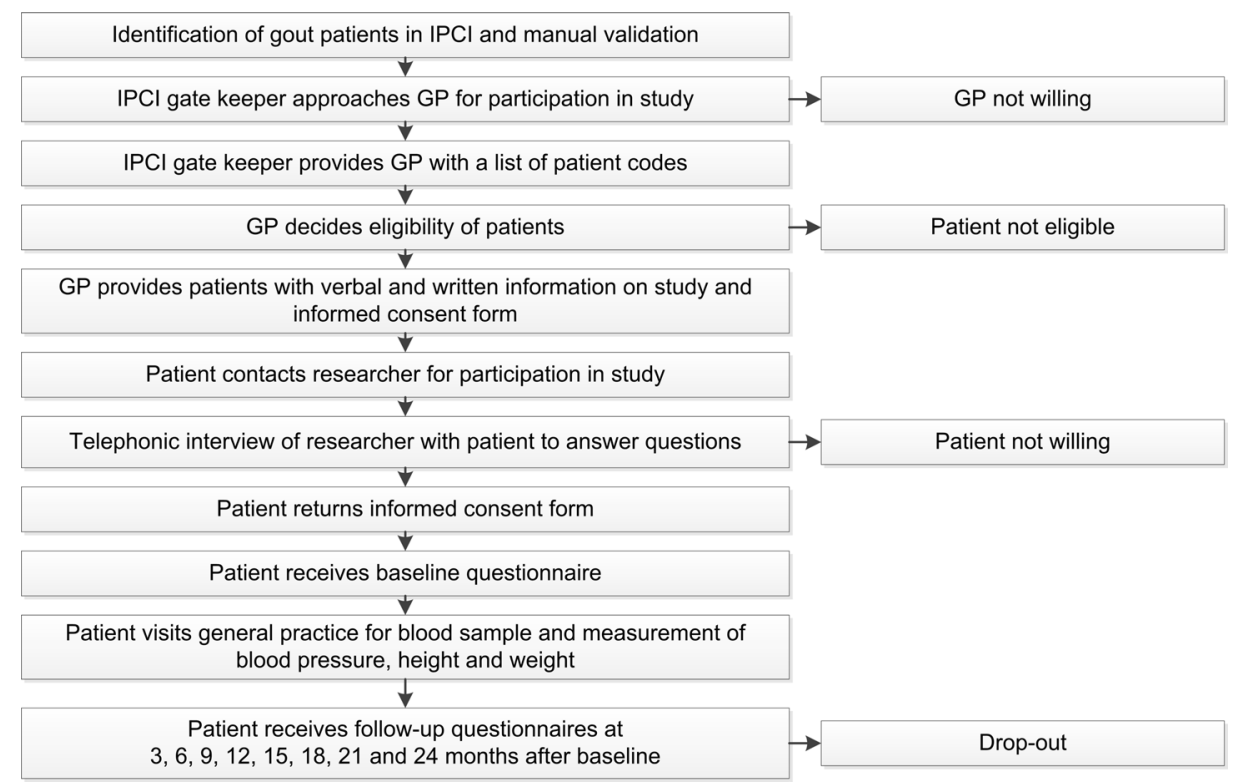

Figure 1 Timeline and study procedures. GP, general practitioner; IPCI, Integrated Primary Care Information Database. 
frequency of gout attacks. The covariates will be patient characteristics, physical and mental health, medication use and dietary habits.

\section{Data monitoring}

Participating in the study results in negligible risks for the participating patients. All study centres will be monitored once every year. The monitoring includes study documents and agreements, patient inclusion, consent, compliance and source document verification, verification of all study procedures and the certification of the participating laboratories. All auditing will be independent from the investigators and the sponsor.

\section{Patients and public involvement}

No patients were involved in the development of research questions, design or conduct of this study. The results will be disseminated to all study participants through a digital (or paper if requested) newsletter.

\section{ETHICS AND DISSEMINATION}

The study is registered in the Dutch Trial Register. In case of any changes or modifications to the research protocol, the sponsor and the medical ethical committee will be informed.

The researcher will obtain written informed consent from potential participants before enrolment in the study. A liability insurance is provided covering any harm incurred by patients during the course of the study. However, the study poses no to negligible risk to participating patients. Patients' identity will remain confidential at all times. Each patient will be given a unique code number which will be used on the case report forms and in the database. Only the researchers have access to the file of the names and corresponding code numbers of the patients. Results from the blood samples are reported via fax machine and stored digitally, all other information from enrolled participants will be collected using paper or digital questionnaires and will be digitally stored in a password-protected environment. Only the executive researcher, project leader and principal investigator have access to the final trial dataset. There are no contractual agreements with the study sponsor that limit access for the investigators. The generated knowledge by this study will be transferred to the Dutch College of General Practitioners (NHG) and other disciplines treating patients with gout including rheumatologists. The study will provide important information about risk factors and treatment of gout in general practice, when updating the clinical NHG guideline M90 'Arthritis' in the future. For this purpose, we will approach the NHG Steering Committee actively with our results. We will also report the results to the Dutch Arthritis Foundation and to the Dutch Association for Rheumatology. In addition to the usual international peer-review publications of the results, we will publish the results of the study in national journals distributed among GPs and other relevant disciplines. Also, we will report the results at (inter)national congresses, and during regional meetings for involved disciplines. No data sharing arrangements have been made, and there are no publication restrictions by the study sponsor.

Acknowledgements The authors would like to thank participating study centres, general practitioners and patients for their support of this study. The authors thank the research assistants who have supported this study.

Contributors KDBvL, SMAB-Z and PJEB designed the study with input from al the authors. KDBvL drafted the manuscript under supervision of AMB, SMAB-Z and PJEB. AMB, MLJ, JvDL, HJEMJ and ARK significantly contributed to the establishment and refinement of study procedures and critically revised the manuscript. All authors approved the final version of the manuscript.

Funding This work was supported by the Netherlands Organisation for Health Research and Development (Zonmw, grant number 80-83910-98-13051).

Competing interests SMAB-Z is consultant for Infirst Healthcare. All other authors declare that they have no competing interests

Patient consent Not required.

Ethics approval Medical Ethical Committee, Erasmus MC, Rotterdam, the Netherlands.

Provenance and peer review Not commissioned; externally peer reviewed.

Open access This is an open access article distributed in accordance with the Creative Commons Attribution Non Commercial (CC BY-NC 4.0) license, which permits others to distribute, remix, adapt, build upon this work non-commercially, and license their derivative works on different terms, provided the original work is properly cited, appropriate credit is given, any changes made indicated, and the use is non-commercial. See: http://creativecommons.org/licenses/by-nc/4.0/.

\section{REFERENCES}

1. Janssens HJ, Lagro HA, van Peet PG, et al. Clinical guideline gout (In Dutch).. Huisarts Wet 2009;9:439-53.

2. Staessen J. The determinants and prognostic significance of serum uric acid in elderly patients of the European Working Party on High Blood Pressure in the Elderly trial. Am J Med 1991;90:50S-4.

3. Seth R, Kydd AS, Buchbinder R, et al. Allopurinol for chronic gout. Cochrane Database Syst Rev 2014;10:CD006077.

4. Roddy E, Mallen CD, Doherty M. Gout. BMJ 2013;347:f5648.

5. Qaseem A, Harris RP, Forciea MA. Management of acute and recurrent gout: a clinical practice guideline from the american college of physicians. Ann Intern Med 2017;166:58-68.

6. Choi HK, Atkinson K, Karlson EW, et al. Purine-rich foods, dairy and protein intake, and the risk of gout in men. $N$ Engl J Med 2004;350:1093-103.

7. Choi HK, Liu S, Curhan G. Intake of purine-rich foods, protein, and dairy products and relationship to serum levels of uric acid: the Third National Health and Nutrition Examination Survey. Arthritis Rheum 2005;52:283-9.

8. Hueskes BA, Roovers EA, Mantel-Teeuwisse AK, et al. Use of diuretics and the risk of gouty arthritis: a systematic review. Semin Arthritis Rheum 2012;41:879-89.

9. Kuo CF, Grainge MJ, Mallen C, et al. Comorbidities in patients with gout prior to and following diagnosis: case-control study. Ann Rheum Dis 2016;75:210-7.

10. van der Lei J, Duisterhout JS, Westerhof HP, et al. The introduction of computer-based patient records in The Netherlands. Ann Intern Med 1993;119:1036-41.

11. Lamberts $\mathrm{H}$, Wood M, Hofmans-Okkes IM. International primary care classifications: the effect of fifteen years of evolution. Fam Pract 1992;9:330-9.

12. Janssens HJ, Fransen J, van de Lisdonk EH, et al. A diagnostic rule for acute gouty arthritis in primary care without joint fluid analysis. Arch Intern Med 2010;170:1120-6.

13. Neogi T, Jansen TL, Dalbeth N, et al. Gout classification criteria: an American College of Rheumatology/European League Against Rheumatism collaborative initiative. Ann Rheum Dis 2015;74:1789-98.

14. Spaetgens B, van der Linden S, Boonen A. The gout assessment questionnaire 2.0: cross-cultural translation into dutch, aspects of validity and linking to the international classification of functioning, disability and health. Rheumatology 2014;53:678-85.

15. Taylor WJ. Gout measures: Gout Assessment Questionnaire (GAQ, GAQ2.0), and physical measurement of tophi. Arthritis Care Res 2011;63:S59-S63. 
16. Colwell HH, Hunt BJ, Pasta DJ, et al. Gout Assessment Questionnaire: Initial results of reliability, validity and responsiveness. Int J Clin Pract 2006;60:1210-7.

17. Aati O, Taylor WJ, Siegert RJ, et al. Development of a patientreported outcome measure of tophus burden: the Tophus Impact Questionnaire (TIQ-20). Ann Rheum Dis 2015;74:2144-50.

18. Sangha O, Stucki G, Liang MH, et al. The Self-Administered Comorbidity Questionnaire: a new method to assess comorbidity for clinical and health services research. Arthritis Rheum 2003;49:156-63.

19. Ware JE, Sherbourne CD. The MOS 36-item short-form health survey (SF-36). I. Conceptual framework and item selection. Med Care 1992;30:473-83.

20. Brooks RG, Jendteg S, Lindgren B, et al. EuroQol: health-related quality of life measurement. Results of the Swedish questionnaire exercise. Health Policy 1991;18:37-48.

21. Craig CL, Marshall AL, Sjöström M, et al. International physical activity questionnaire: 12-country reliability and validity. Med Sci Sports Exerc 2003;35:1381-95.
22. Svarstad BL, Chewning BA, Sleath BL, et al. The Brief Medication Questionnaire: a tool for screening patient adherence and barriers to adherence. Patient Educ Couns 1999;37:113-24.

23. Siebelink E, Geelen A, de Vries JH. Self-reported energy intake by FFQ compared with actual energy intake to maintain body weight in 516 adults. Br J Nutr 2011;106:274-81.

24. Goldbohm RA, van 't Veer P, van den Brandt PA, et al. Reproducibility of a food frequency questionnaire and stability of dietary habits determined from five annually repeated measurements. Eur J Clin Nutr 1995;49:420-9.

25. Goldbohm RA, van den Brandt PA, Brants HA, et al. Validation of a dietary questionnaire used in a large-scale prospective cohort study on diet and cancer. Eur J Clin Nutr 1994;48:253-65.

26. Feunekes GI, Van Staveren WA, De Vries JH, et al. Relative and biomarker-based validity of a food-frequency questionnaire estimating intake of fats and cholesterol. Am J Clin Nutr 1993;58:489-96. 